\title{
Comparable efficacy of endoscopic transpapillary gallbladder drainage and percutaneous transhepatic gallbladder drainage in acute cholecystitis
}

\section{(ㄷ)(1) $(2) \Theta$}

\author{
Authors \\ Chikara lino 1,2,3, Tadashi Shimoyama ${ }^{3}$, Takasato Igarashi' ${ }^{2,3}$, Tomoyuki Aihara², Kentaro Ishii², Juichi Sakamoto², \\ Hiroshi Tono ${ }^{2}$, Shinsaku Fukuda ${ }^{3}$
}

Institutions

1 Department of Gastroenterology and Hematology, Hirosaki National Hospital, Aomori, Japan

2 Department of Internal medicine, Hirosaki Municipal Hospital, Aomori, Japan

3 Department of Gastroenterology, Hirosaki University Graduate School of Medicine, Aomori, Japan

submitted 19.5.2017

accepted after revision 22.1.2018

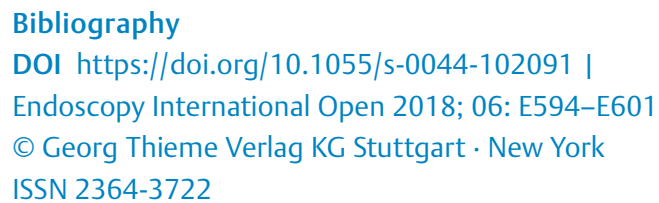

Corresponding author

Chikara lino, Department of Gastroenterology and Hematology, Hirosaki National Hospital, 1, Tominocho, Oaza, Hirosaki, Aomori 036-8545, Japan

Fax: +81-172-33-8614

chikaran0601@yahoo.co.jp

\section{ABSTRACT}

Background and study aims Although endoscopic transpapillary gallbladder drainage (ETGBD) is reportedly useful in patients who have acute cholecystitis, its efficacy has not been compared to that of percutaneous transhepatic gallbladder drainage (PTGBD). We retrospectively compared the efficacy and safety of ETGBD and PTGBD in patients with acute cholecystitis.

Patients and methods We studied 75 patients who required gallbladder drainage for acute cholecystitis between January 2014 and December 2016. Using propensity score matching analysis, we compared the clinical efficacy and length of hospitalization in patients successfully treated with ETGBD and PTGBD. Moreover, we assessed the predictive factors for hospitalization period $<30$ days using multivariate analysis.

Results ETGBD and PTGBD were successfully performed in 33 patients $(77 \%)$ and 42 patients $(100 \%)(P<0.001)$. Twenty-seven matched pairs were obtained after propensity score matching analysis. No significant differences were observed between patients treated with ETGBD and those treated with PTGBD with respect to improvement in white blood cell count and serum C-reactive protein level. The length of hospitalization in patients treated with ETGBD was significantly shorter than in those treated with PTGBD regardless of the need for surgery. Multivariate logistic regression analysis revealed ETGBD (odds ratio, 7.07; 95\% confidence interval 2.22-22.46) and surgery (odds ratio 0.26 ; $95 \%$ confidence interval $0.09-0.79$ ) as independent factors associated with hospitalization period. There were no significant differences in occurrence of complications in ETGBD and PTGBD procedure.

Conclusions ETGBD was shown to be as useful as PTGBD for treatment of acute cholecystitis and was associated with shorter hospitalization period. ETGBD can be an alternative treatment option for acute cholecystitis at times when PTGBD is not possible.

\section{Introduction}

Acute cholecystitis is a common gastrointestinal emergency, for which urgent cholecystectomy is the standard treatment $[1,2]$. Although cholecystectomy is generally a safe procedure, the mortality rate is higher in critically ill or elderly patients [3, 4]. According to Tokyo guidelines 2013, early gallbladder drain- age is recommended for patients with severe local inflammation and/or severe (Grade III) acute cholecystitis [2].

Percutaneous transhepatic gallbladder drainage (PTGBD) is a widely performed and established method for drainage of the gallbladder [5]. However, it cannot be performed in patients with thrombocytopenia, coagulation disorders, massive ascites, or those taking anticoagulants or antiplatelet drugs. 


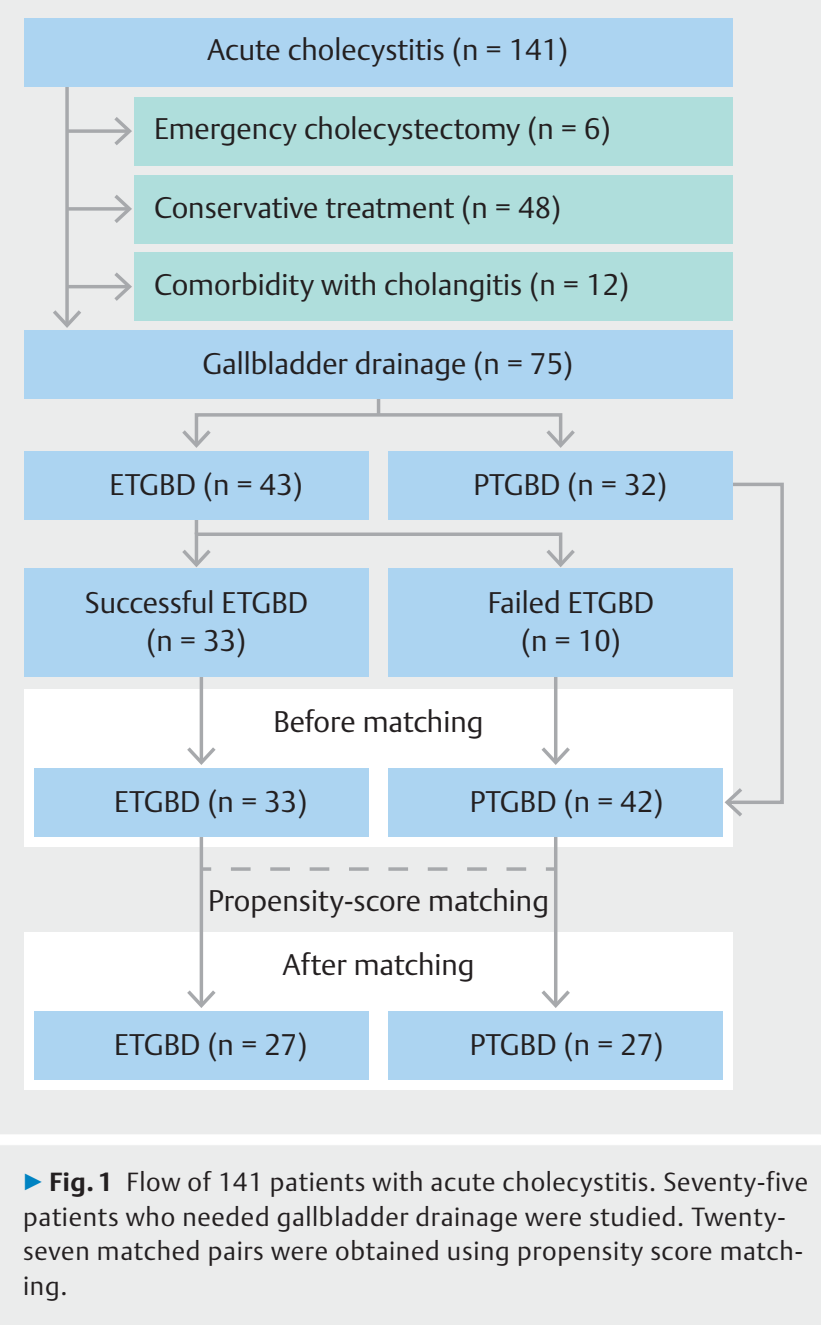

There are several reports on the usefulness and safety of endoscopic transpapillary gallbladder drainage (ETGBD) including endoscopic nasogallbladder drainage (ENGBD) [6-13] and endoscopic gallbladder stenting (EGBS) in patients with acute cholecystitis [14-21]. However, there are few studies comparing the efficacy of ETGBD and PTGBD in the treatment of acute cholecystitis.

We performed a retrospective study, using propensity score matching analysis, to compare the usefulness and safety of ETGBD and PTGBD in patients with acute cholecystitis. Moreover, we analyzed predictive factors for shorter hospitalization using multivariate analysis.

\section{Patients and methods}

\section{Patients}

Between January 2014 and December 2016, 141 consecutive patients with acute cholecystitis who were admitted to Hirosaki Municipal Hospital, Aomori, Japan were studied. Of these, we excluded six patients who underwent emergency cholecystectomy, 12 with comorbidity with cholangitis and 48 who received conservative treatment with antibiotics from the study
- Table 1 Characteristics of gallbladder drainage.

\begin{tabular}{|c|c|c|}
\hline & ETGBD $(n=43)$ & PTGBD $(n=32)$ \\
\hline \multicolumn{3}{|l|}{ Criteria } \\
\hline - Antiplatelet agents & 7 & 0 \\
\hline - Anticoagulant agents & 5 & 0 \\
\hline - Thrombocytopenia & 2 & 0 \\
\hline - Bleeding tendency & 1 & 0 \\
\hline - Ascites & 4 & 0 \\
\hline - Choledocholithiasis & 11 & 0 \\
\hline - Chilaiditi syndrome & 2 & 0 \\
\hline - Dementia & 19 & 4 \\
\hline History of gastrectomy & 0 & 5 \\
\hline \multicolumn{3}{|l|}{ Comorbidities } \\
\hline - Cerebrovascular disease & 4 & 6 \\
\hline - Ischemic heart disease & 2 & 2 \\
\hline - Renal failure & 2 & 1 \\
\hline Advanced malignancy & 5 & 4 \\
\hline \multicolumn{3}{|c|}{$\begin{array}{l}\text { ETGBD, endoscopic transpapillary gallbladder drainage; PTGBD, percuta- } \\
\text { neous transhepatic gallbladder drainage. }\end{array}$} \\
\hline
\end{tabular}

( $\triangleright$ Fig. 1). We evaluated 75 patients who needed gallbladder drainage. As initial therapy, 43 patients and 32 patients underwent ETGBD and PTGBD, respectively. ETGBD was selected for patients who used antiplatelet agents or anticoagulant agents, and had thrombocytopenia, bleeding tendency, ascites, suspicious of choledocholithiasis, Chilaiditi syndrome and dementia with a risk of self-removal of the drainage tube. All 43 patients who underwent ETGBD met the criteria. There were seven patients who used antiplatelet agents, five who used anticoagulant agents, three who had thrombocytopenia and bleeding tendency, four with ascites, 11 with choledocholithiasis, two patients with Chilaiditi syndrome and 19 patients with dementia ( $\triangleright$ Table 1$)$. There were also four patients with dementia. These patients underwent PTGBD first in spite of preprocedural risk, because they had previous histories of gastrectomy. When ETGBD had technically failed, PTGBD was performed. Patients who were successfully treated with ETGBD were compared to those treated with PTGBD, including those who had initially failed ETCBD. Moreover, the clinical courses of the two groups were compared using propensity score-matched analysis.

Patients were diagnosed as having acute cholecystitis based on the following criteria: clinical symptoms of right upper quadrant and/or epigastric pain or tenderness; signs of systemic inflammation including fever and high WBC count or high levels of CRP; and positive findings associated with distended gallbladder, thickening of the wall of gallbladder or, fluid around the gallbladder, as confirmed on abdominal ultrasonography or computed tomography [22]. 

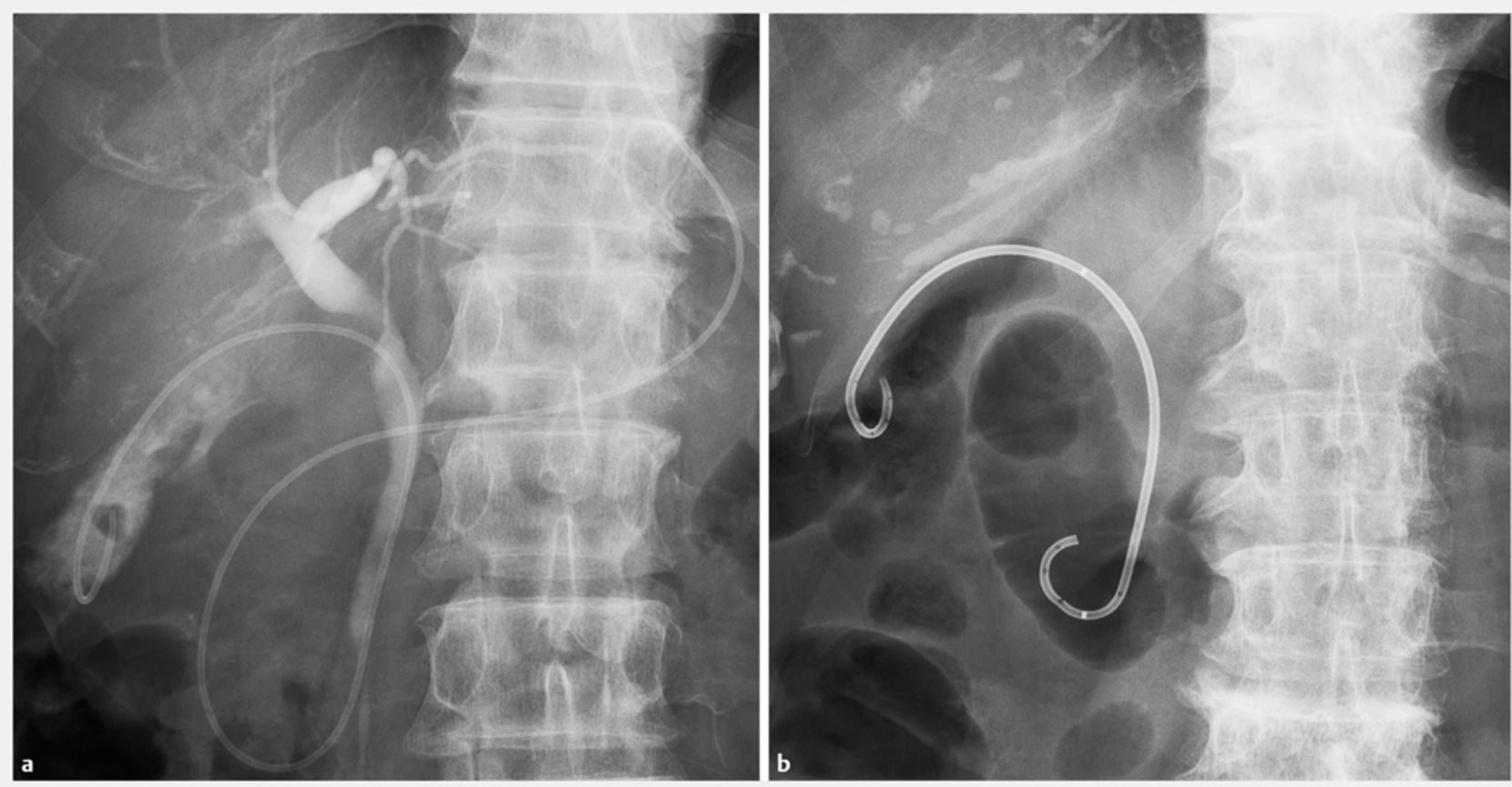

Fig. 2 X-ray images of a endoscopic nasogallbladder drainage and $\mathbf{b}$ endoscopic gallbladder stenting.

\section{ETGBD}

Following endoscopic retrograde cholangiopancreatography (ERCP), a 0.035-inch Radifocus guidewire (Terumo, Tokyo, Japan) was inserted into the cystic duct and advanced into the gallbladder. After successful cannulation in the gallbladder, a 5-French pigtail-type nasobiliary drainage tube (Hanako, Tokyo, Japan) was inserted into it, and subsequently, the gallbladder was drained and rinsed with a saline solution through the tube until a completely clear fluid was obtained ( $\triangleright$ Fig. 2a). In some cases, because the nasobiliary drainage tube has a high risk of dislodgement, it was replaced with a 7-French double-pigtail stent $(15 \mathrm{~cm}$, Olympus Medical Systems, Tokyo, Japan) ( Fig. 2b).

\section{PTGBD}

PTGBD was performed using the Seldinger technique. After performing ultrasound-guided transhepatic gallbladder puncture using an 18-gauge needle, a guidewire was inserted into the gallbladder which was followed by insertion of a 7 to 9 French pigtail catheter using a guidewire under fluoroscopy.

\section{Propensity score matching}

To confirm the validity of this retrospective analysis, we used propensity score matching method to compare the clinical course of patients treated successfully with ETGBD and PTGBD. Propensity scores were calculated using logistic regression analysis. Age, sex and severity of acute cholecystitis were used as matching factors and included in a multivariable logistic regression analysis. Based on the scores obtained for each pa- tient, patients treated with ETGBD were matched to patients treated with PTGBD using calipers of width equal to 0.2 of the standard deviation (SD) of the logit of the propensity score.

\section{Outcome}

Study outcomes were success rates and clinical efficacy of ETGBD and PTGBD procedures. Success rate was defined based on successful placement of the catheter into the gallbladder. Clinical efficacy was evaluated based on WBC count and CRP levels at 7 days after treatment, time required for normalization of WBC count, time required for serum CRP levels to decrease below $1.0 \mathrm{mg} / \mathrm{dL}$, and duration of hospitalization. Moreover, to elucidate factors associated with hospitalization $<30$ days, we performed univariate analysis and then multivariate logistic regression analysis. Univariate analysis was performed with gender, age, gallbladder drainage (ETGBD or PTGBD), surgery, serum levels of WBC, serum levels of CRP, severity grade of cholecystitis, use of antiplatelet drugs, use of anticoagulant drugs, complications of ascites, having a common bile duct stone, having a gallbladder stone and having dementia. Variables with $P<0.1$ in the univariate analysis were included in the multivariate analysis using logistic regression.

\section{Ethics statement}

This study was performed in accordance with the ethical standards of the Declaration of Helsinki, and approved by the institutional review board at Hirosaki Municipal Hospital (no. 996). All patients or family members (if the patient had dementia) had provided written consent. 
- Table 2 Technical success and adverse events between ETGBD and PTGBD.

\begin{tabular}{|l|l|l|l|}
\hline & ETGBD & PTGBD & P value \\
\hline First gallbladder drainage & $\mathrm{n}=43$ & $\mathrm{n}=32$ & \\
\hline Technical success & $33(77 \%)$ & $32(100 \%)$ & 0.004 \\
\hline Adverse event & & & \\
\hline - Pancreatitis & $2(4.7 \%)$ & - & \\
\hline - Cystic duct injury & $2(4.7 \%)$ & - & \\
\hline - Bile leak & - & $2(6.3 \%)$ & \\
\hline PTGBD as a crossover from & & $\mathrm{n}=10$ & \\
\hline ETGBD & - & $10(100 \%)$ & \\
\hline Technical success & & & \\
\hline Adverse event & & & \\
\hline ETGBD, endoscopic transpapillary gallbladder drainage; PTGBD, percuta- \\
\hline neous transhepatic gallbladder drainage.
\end{tabular}

Table 3 Patient characteristics.

\section{Statistical analysis}

Statistical analyses of the clinical data were performed using JMP ver. 12.1 (SAS Institute, Cary, NC). Categorical variables are shown as frequencies and percentages and continuous variables are shown as mean with SD or median with interquartile range. Categorical variables were compared using the chisquare test or the Fisher's exact test and continuous variables were compared using Mann-Whitney U-test. After propensity score matching, categorical variables were compared using the McNemar's test and continuous variables were compared using the Wilcoxon's matched-pairs signed-rank test. A $P$ value $<0.05$ was considered significant for all tests.

\section{Results}

ETGBD was successfull in 33 patients (77\%) ( $\bullet$ Table 2). PTGBD was successfull in 10 patients who in whom ETGBD had failed as well as in 32 patients who had received PTGBD as the initial therapy (100\%). The success rate for PTGBD was significantly higher for ETGBD $(P<0.001)$. In 10 patients with failure of ETGBD, there were seven cases with failure to detect the cystic duct, three cases without passage of the guidewire into the cystic duct due to stricture caused by severe inflammation or the gallstones in the cystic duct. Preprocedural clinical data on these 10 patients were similar to those for other patients with successful ETGBD. Mild acute pancreatitis after ETGBD occurred in two patients. Cystic duct injury during ETGBD procedure occurred in two patients. On the other hand, bile leak related to

\begin{tabular}{|c|c|c|c|c|c|c|}
\hline & \multicolumn{3}{|c|}{ Before matching } & \multicolumn{3}{|c|}{ After matching } \\
\hline & ETGBD & PTGBD & $P$ value & ETGBD & PTGBD & $P$ value \\
\hline & $(n=33)$ & $(n=42)$ & & $(n=27)$ & $(n=27)$ & \\
\hline Male, n (\%) & $15(45.4)$ & $26(61.9)$ & 0.155 & $15(55.6)$ & $13(48.1)$ & 0.414 \\
\hline Age mean \pm SD (years) & $77.9 \pm 12.6$ & $73.5 \pm 13.5$ & 0.088 & $76.7 \pm 13.1$ & $75 \pm 13.2$ & 0.689 \\
\hline \multicolumn{7}{|l|}{ Severity grade of cholecystitis } \\
\hline - Mild/moderate/severe & $9 / 19 / 5$ & $13 / 21 / 8$ & 0.800 & $8 / 14 / 5$ & $7 / 15 / 5$ & 0.951 \\
\hline $\mathrm{WBC}$ mean $\pm \mathrm{SD}\left(\times 10^{3} / \mu \mathrm{L}\right)$ & $13.1 \pm 5.4$ & $13.7 \pm 4.6$ & 0.408 & $12.5 \pm 5.3$ & $13.7 \pm 5.2$ & 0.285 \\
\hline $\mathrm{CRP}$ mean $\pm \mathrm{SD}(\mathrm{mg} / \mathrm{dL})$ & $15.8 \pm 9.7$ & $19.9 \pm 8.0$ & 0.034 & $15.5 \pm 9.5$ & $19.8 \pm 8.9$ & 0.127 \\
\hline Antiplatelet drugs, n (\%) & $7(21.2)$ & $0(0)$ & 0.002 & $6(22.2)$ & $0(0)$ & 0.041 \\
\hline Anticoagulant drugs, n (\%) & $4(12.1)$ & $1(2.4)$ & 0.162 & $3(11.1)$ & $0(0)$ & 0.248 \\
\hline Ascites, n (\%) & $4(12.1)$ & $0(0)$ & 0.034 & $2(7.4)$ & $0(0)$ & 0.479 \\
\hline Common bile duct stone, $\mathrm{n}(\%)$ & $7(21.2)$ & $4(9.5)$ & 0.197 & $5(18.5)$ & $3(11.1)$ & 0.683 \\
\hline Dementia, n (\%) & $14(42.4)$ & $9(21.4)$ & 0.076 & $11(40.7)$ & $8(29.6)$ & 0.546 \\
\hline Gallbladder stone, n (\%) & $27(81.8)$ & $35(83.3)$ & 0.904 & $23(85.1)$ & $22(81.4)$ & 1.000 \\
\hline ENGBD/EGBD & $20 / 13$ & - & - & $17 / 10$ & - & - \\
\hline
\end{tabular}

CRP, C-reactive protein; EGBS, endoscopic gallbladder drainage; ENGBD, endoscopic nasogallbladder drainage; ETGBD, endoscopic transpapillary gallbladder drainage; PTGBD, percutaneous transhepatic gallbladder drainage; SD, standard deviation; WBC, white blood cells. 
- Table 4 Patient characteristics after gallbladder drainage.

\begin{tabular}{|c|c|c|c|c|c|c|}
\hline & \multicolumn{3}{|c|}{ Before matching } & \multicolumn{2}{|c|}{ After matching } & \multirow[b]{2}{*}{$P$ value } \\
\hline & ETGBD & PTGBD & $P$ value & ETGBD & PTGBD & \\
\hline & $(n=33)$ & $(n=42)$ & & $(n=27)$ & $(n=27)$ & \\
\hline WBC 7 days after the treatment $\left(\times 10^{3} / \mu \mathrm{L}\right)$ & $6.90 \pm 1.83$ & $7.78 \pm 2.46$ & 0.063 & $6.80 \pm 1.80$ & $7.75 \pm 2.81$ & 0.149 \\
\hline CRP 7 days after treatment $(\mathrm{mg} / \mathrm{dL})$ & $3.25 \pm 4.61$ & $4.65 \pm 4.70$ & 0.070 & $3.28 \pm 5.02$ & $4.93 \pm 5.07$ & 0.080 \\
\hline - Period until normalization of WBC (days) & 5 & 7 & 0.170 & 5 & 7 & 0.149 \\
\hline $\mathrm{CRP}<1.0 \mathrm{mg} / \mathrm{dL}$ at discharge, $\mathrm{n}(\%)$ & 31 (93.9) & $38(90.5)$ & 0.583 & $26(96.3)$ & $25(92.6)$ & 0.479 \\
\hline - Period until $C R P<1.0 \mathrm{mg} / \mathrm{dL}$ (days) & 10 & 14 & 0.047 & 10 & 14 & 0.197 \\
\hline Without surgery, $\mathrm{n}(\%)$ & $18(54.6)$ & $20(47.6)$ & 0.552 & $14(51.9)$ & $13(48.1)$ & 0.773 \\
\hline - Length of hospitalization (days) & $15(10-29)$ & $32(26-49)$ & $<0.001$ & $15(10-31)$ & $32(26-52)$ & 0.003 \\
\hline Received surgery, n (\%) & $15(45.4)$ & $22(52.4)$ & 0.551 & $13(48.1)$ & $14(51.9)$ & 0.773 \\
\hline - Length of hospitalization (days) & $29(24-37)$ & $47(33-67)$ & $<0.001$ & $27(23-37)$ & $47(37-61)$ & 0.005 \\
\hline Complications, n (\%) & $4(12.1)$ & $2(4.8)$ & 0.201 & $4(14.8)$ & $2(7.4)$ & 0.683 \\
\hline - Pancreatitis after ETGBD, n (\%) & $2(6.0)$ & - & - & $2(7.4)$ & - & - \\
\hline - Cystic duct injury, n (\%) & $2(6.0)$ & - & - & $2(7.4)$ & - & - \\
\hline - Bile leak, n (\%) & - & $2(4.8)$ & - & - & $2(7.4)$ & - \\
\hline
\end{tabular}

PTGBD occurred in two patients. There were no tube-related problems in any patients after ETGBD or PTGBD.

Overall, this study included 33 patients in the ETGBD and 42 patients in the PTGBD group. There were significant differences in initial CRP levels between the two groups ( $\bullet$ Table 3 ). After treatment, time required to attain CRP level $<1.0 \mathrm{mg} / \mathrm{dL}$ and length of hospitalization with and without surgery in the ETCBD group were significantly shorter than those in the PTGBD group $(P=0.047, P<0.001$ and $P<0.001$, respectively) ( $\vee$ Table 4$)$.

Twenty-seven matched pairs were obtained after propensity score matching. No significant differences with respect to patients' background were observed in the two groups. There were no significant differences in the groups with respect to WBC count and serum levels of CRP at 7 days after treatment or in time required to attain normal WBC count and CRP level $<1.0 \mathrm{mg} / \mathrm{dL}$. Length of hospitalization in the ETCBD group was significantly shorter than that in the PTCBD group $(P=0.003$ and $P=0.005$ ) regardless of the need for surgery.

Results of univariate analysis for each factor for hospitalization $<30$ days are shown in $>$ Table $\mathbf{5}$. The comparison between the two groups in univariate analysis showed that variables with $P<0.1$ were gallbladder drainage, surgery and use of anticoagulant drugs. Results of the multivariate logistic regression analysis revealed that both ETGBD (odds ratio, 7.07; $95 \%$ confidence interval 2.22-22.46, $P<0.001$ ) and surgery (odds ratio, 0.26; $95 \%$ confidence interval $0.09-0.79, P=0.018)$ were independent factors for hospitalization $<30$ days ( $\triangleright$ Table 6 ).

\section{Discussion}

Our study using propensity score matching analysis demonstrated that ETGBD was as useful as PTGBD for treatment of cholecystitis and successful ETGBD resulted in a shorter hospital stay compared to PTGBD. Furthermore, multivariate logistic regression analysis revealed that ETGBD was positively and surgery was negatively and independently associated with hospitalization $<30$ days. These results suggest that ETCBD was as effective as PTGBD for management of inflammation associated with acute cholecystitis. There were no significant differences between ETGBD and PTGBD with respect to decrease in WBC count and serum CRP levels after gallbladder drainage. Reportedly, ETGBD is a practical and effective alternative to PTGBD [6-21]. However, it has not been established as a standard treatment owing to technical difficulty [5]. Our study revealed technical success rates of $77 \%$ and $100 \%$, respectively, for ETGBD and PTGBD. That was similar to results from previous studies, which reported that the technical success rate of ETGBD varied from $64 \%$ to $100 \%$ [ $6-21]$. Failure of ETGBD was attributable to failure to detect the cystic duct and pass the guidewire into it due to a stricture caused by severe inflammation and gallstones in the cystic duct. Previous reports have demonstrated a $100 \%$ technical success rate with PTGBD, suggesting that the procedure is not difficult [23]. Therefore, PTGBD has been established as the standard procedure for gallbladder drainage. However, PTGBD is contraindicated in some patients, including those using antiplatelet agents or anticoa- 
- Table 5 Univariate analysis of factors for hospitalization $<30$ days.

\begin{tabular}{|c|c|c|c|c|c|}
\hline Variable & & $\mathbf{n}$ & OR & (95\%Cl) & $P$ value \\
\hline \multirow[t]{2}{*}{ Gender } & Male & 41 & 0.97 & $(0.39-2.42)$ & 0.951 \\
\hline & Female & 34 & 1 & & \\
\hline \multirow[t]{2}{*}{ Age (years) } & $\geq 80$ & 39 & 1.83 & $(0.73-4.60)$ & 0.196 \\
\hline & $<80$ & 36 & 1 & & \\
\hline \multirow[t]{2}{*}{ Drainage } & ETGBD & 33 & 7.52 & $(2.68-21.04)$ & $<0.001$ \\
\hline & PTGBD & 42 & 1 & & \\
\hline \multirow[t]{2}{*}{ Surgery } & Yes & 37 & 0.31 & $(0.12-0.81)$ & 0.016 \\
\hline & No & 38 & 1 & & \\
\hline \multirow[t]{2}{*}{$\mathrm{WBC}\left(\times 10^{3} / \mu \mathrm{L}\right)$} & $\geq 12.0$ & 41 & 0.63 & $(0.25-1.57)$ & 0.322 \\
\hline & $<12.0$ & 34 & 1 & & \\
\hline \multirow[t]{2}{*}{ CRP (mg/dL) } & $\geq 15$ & 46 & 0.72 & $(0.28-1.82)$ & 0.486 \\
\hline & $<15$ & 29 & 1 & & \\
\hline \multicolumn{6}{|c|}{ Severity of cholecystitis } \\
\hline - Severe & & 13 & 0.67 & $(0.20-2.27)$ & 0.516 \\
\hline - Moderate & & 40 & 1.07 & $(0.43-2.67)$ & 0.871 \\
\hline " Mild & & 22 & 1 & & \\
\hline \multirow[t]{2}{*}{ Antiplatelet drugs } & Yes & 7 & 8.07 & $(0.92-70.73)$ & 0.059 \\
\hline & No & 68 & 1 & & \\
\hline \multirow[t]{2}{*}{ Anticoagulants } & Yes & 5 & 5.03 & $(0.53-47.34)$ & 0.158 \\
\hline & No & 70 & 1 & & \\
\hline \multirow[t]{2}{*}{ Ascites } & Yes & 4 & 1.15 & $(0.15-8.63)$ & 0.891 \\
\hline & No & 71 & 1 & & \\
\hline \multirow[t]{2}{*}{ CBD stone } & Yes & 11 & 1.45 & $(0.40-5.23)$ & 0.572 \\
\hline & No & 64 & 1 & & \\
\hline \multirow[t]{2}{*}{ Gallbladder stone } & Yes & 62 & 1.50 & $(0.44-5.10)$ & 0.516 \\
\hline & No & 13 & 1 & & \\
\hline \multirow[t]{2}{*}{ Dementia } & Yes & 23 & 0.64 & $(0.24-1.74)$ & 0.386 \\
\hline & No & 52 & 1 & & \\
\hline
\end{tabular}

gulants or who have thrombocytopenia, bleeding tendency, severe ascites, anatomic abnormalities, or dementia with a risk of self-removal of the drainage tube. ETGBD was as effective as PTGBD for treatment of acute cholecystitis. Although it is unsuccessful in some cases, ETGBD is an effective alternative in patients for whom PTGBD cannot be performed. Therefore, ETGBD should be one of the considerable treatment options for acute cholecystitis.

A recent meta-analysis showed that ETGBD archived a similar technical success rate as PTGBD but appeared to be safer than PTGBD in sub-analysis [24]. However, in that meta-analysis, only three of 23 studies had compared the efficacy of
ETGBD and PTGBD. In two of three studies, ETGBD included endoscopic ultrasound (EUS)-guided transmural stenting [25, 26]. In another study, ETGBD was directly compared with PTGBD, but the outcome measured in that research was rate of recurrence of cholecystitis and ETGBD and PTGBD were performed in different periods [19]. Therefore, our study is the first study to compare the efficacy of ETGBD and PTGBD in patients with acute cholecystitis in the same period.

Our results showed that ETGBD procedure resulted into a shorter hospital stay than that did PTGBD in propensity score matching analysis. Rates of surgery observed after ETGBD were almost the same as those after PTGBD. In patients both 
- Table 6 Multivariate analysis of factors for hospitalization $<30$ days.

\begin{tabular}{|l|l|l|l|}
\hline Variable & OR & $\mathbf{9 5 \% C l}$ & P value \\
\hline Drainage & \multicolumn{3}{|l|}{} \\
\hline - ETGBD & 7.07 & $(2.22-22.46)$ & $<0.001$ \\
\hline - PTGBD & 1 & & \\
\hline Surgery & & & \\
\hline - Yes & 0.26 & $(0.09-0.79)$ & 0.018 \\
\hline - No & 1 & & \\
\hline Anticoagulant drugs & & & \\
\hline - Yes & 2.74 & $(0.26-28.56)$ & 0.399 \\
\hline $\begin{array}{l}\text { - No } \\
\text { Cl, Confidence Interval; ETGBD, endoscopic transpapillary gallbladder }\end{array}$ \\
\hline $\begin{array}{l}\text { drainage; OR, odds ratio; PTGBD, percutaneous transhepatic gallbladder } \\
\text { drainage. }\end{array}$
\end{tabular}

with and without the need for surgery, length of hospitalization after ETGBD was shorter than that after PTGBD. Moreover, ETGBD was an independent factor for shorter hospitalization. The tube inserted during PTGBD cannot be pulled out until a fistula has formed, which requires at least 2 weeks [27]. Therefore, patients who underwent ETGBD were eligible for discharge earlier than those who underwent PTGBD. ETGBD is a less invasive procedure than PTGBD because there is no need for skin puncture and the hospital stay is shorter.

All the patients treated with ETGBD and PTGBD showed a decrease in WBC count and serum levels of CRP after gallbladder drainage. Although WBC count normalized in all patients, serum levels of CRP had not decreased below $1.0 \mathrm{mg} / \mathrm{dL}$ in six patients at time of discharge. However, their serum CRP levels were under $2.0 \mathrm{mg} / \mathrm{dL}$, and they had no clinical symptoms. Of these patients, two and four patients underwent ETGBD and PTGBD, respectively. Although there were no significant differences between ETGBD and PTGBD in terms of improvement of inflammation, patients who underwent PTGBD had higher WBC counts and CRP levels than those who underwent ETGBD. These individuals may have a small amount of bile spillage around the gallbladder due to puncture of the gallbladder as an invasive procedure.

There were no significant differences in incidence of complications between the patients treated with ETGBD and PTGBD. Adverse events associated with ETGBD were mild according to the ASGE workshop definition [28]. None of the complications were severe or affected length of hospitalization. The complications typically associated with ETGBD are post-ERCP pancreatitis, perforation of the cystic duct, and cholangitis. The rate of complications is reported to be $0 \%$ to $16 \%$ [6-21], which is similar to our results. Two patients developed mild pancreatitis after ETGBD. However, their serum amylase levels became normal within 5 days after the procedure. In two patients with cystic duct injury, the guidewire penetrated through the cystic duct wall during ETGBD. One of them was successfully treated with ETGBD, while the other required PTGBD with endoscopic nasobiliary drainage. There was no complaint of abdominal pain after the procedure, computed tomography did not show free air or bile leak, and the WBC count and CRP levels did not increase following the procedure. Complications typically associated with PTGBD are puncture-induced hemorrhage, bile leak, and pneumothorax. Two patients with bile leak associated with PTGBD had mild abdominal pain and were treated conservatively. Although the rate of complications associated with ETGBD was slightly higher than that with PTGBD, the complications were not severe.

ETGBD was not feasible for five patients with a history of gastrectomy because of technical difficulty. In 10 patients, ETGBD had failed due to stricture caused by severe inflammation and the gallstones in the cystic duct resulting in the failure of detection of the cystic duct and insertion of the guidewire into the cystic duct. These patients underwent PTGBD as an alternative drainage. Recently EUS-guided transmural gallbladder drainage has been reported as an effective method for managing acute cholecystitis [5, 23, 24]. A randomized controlled study showed that EUS-guided transmural gallbladder drainage was comparable to PTGBD in feasibility and efficacy [25]. In the current study, especially for patients with dementia and failure of ETGBD, EUS-guided transmural gallbladder drainage may be a better alternative. However, EUS-guided transmural gallbladder drainage is not a standardized procedure and it is currently performed in limited institution that have expertise in both EUS and ERCP.

Our study has several limitations. First, it was a retrospective analysis of a small number of patients from a single institution. Thus, we could not control for selection bias and confounding factors. However, use of propensity score matching enabled us to analyze 27 matched pairs between ETGBD and PTGBD. Furthermore, we performed multivariate logistic regression analysis to compare between ETGBD and PTGBD. Second, our study did not evaluate the effectiveness of ENGBD and EGBS separately. There may be some differences in efficacy and safety between ENGBD and EGBS. However, a recent prospective study has shown that efficacy and safety were almost similar for treatment of acute cholecystitis in patients who cannot undergo emergency cholecystectomy [29].

\section{Conclusion}

In conclusion, we found that ETGBD was comparable to PTGBD in terms of efficacy and safety for treatment of acute cholecystitis. The success rate of ETGBD was not low (77\%). The hospital stay associated with successful ETGBD was shorter than that with PTGBD. These results suggest that ETGBD can be an alternative treatment option for acute cholecystitis at times when PTGBD is not possible.

\section{Competing interests}

None 


\section{References}

[1] Hannan EL, Imperato PJ, Nenner RP et al. Laparoscopic and open cholecystectomy in New York State: mortality, complications, and choice of procedure. Surgery 1999; 125: $223-231$

[2] Yamashita Y, Takada T, Strasberg SM et al. TG13 surgical management of acute cholecystitis. J Hepatobiliary Pancreat Sci 2013; 20: $89-96$

[3] Houghton PW, Jenkinson LR, Donaldson LA. Cholecystectomy in the elderly: a prospective study. $\mathrm{Br}$ J Surg 1985; 72: 220 - 222

[4] Frazee RC, Nagorney DM, Mucha P Jr. Acute acalculous cholecystitis. Mayo Clin Proc 1989; 64: 163 - 167

[5] Tsuyuguchi T, Itoi T, Takada T et al. TG13 indications and techniques for gallbladder drainage in acute cholecystitis. J Hepatobiliary Pancreat Sci 2013; $20: 81-88$

[6] Feretis C, Apostolidis N, Mallas E et al. Endoscopic drainage of acute obstructive cholecystitis in patients with increased operative risk. Endoscopy 1993; 25: $392-395$

[7] Baron TH, Schroeder PL, Schwartzberg MS et al. Resolution of Mirizzi's syndrome using endoscopic therapy. Gastrointest Endosc 1996; 44: $343-345$

[8] Nakatsu T, Okada H, Saito K et al. Endoscopic transpapillary gallbladder drainage (ETGBD) for the treatment of acute cholecystitis. J Hepatobiliary Pancreat Surg 1997; 4: 31 - 35

[9] Toyota N, Takada T, Amano H et al. Endoscopic naso-gallbladder drainage in the treatment of acute cholecystitis: alleviates inflammation and fixes operator's aim during early laparoscopic cholecystectomy. J Hepatobiliary Pancreat Surg 2006; 13: 80 - 85

[10] Kjaer DW, Kruse A, Funch-Jensen P. Endoscopic gallbladder drainage of patients with acute cholecystitis. Endoscopy 2007; 39: $304-308$

[11] Itoi T, Sofuni A, Itokawa F et al. Endoscopic transpapillary gallbladder drainage in patients with acute cholecystitis in whom percutaneous transhepatic approach is contraindicated or anatomically impossible (with video). Gastrointest Endosc 2008; 68: 455 - 460

[12] Ogawa O, Yoshikumi H, Maruoka N et al. Predicting the success of endoscopic transpapillary gallbladder drainage for patients with acute cholecystitis during pretreatment evaluation. Can J Gastroenterol 2008; 22: $681-685$

[13] Yane K, Maguchi H, Katanuma A et al. Feasibility, efficacy, and predictive factors for the technical success of endoscopic nasogallbladder drainage: a prospective study. Gut Liver 2015; 9: 239-246

[14] Tamada K, Seki H, Sato K et al. Efficacy of endoscopic retrograde cholecystoendoprosthesis (ERCCE) for cholecystitis. Endoscopy 1991; 23: $2-3$

[15] Johlin FC Jr, Neil GA. Drainage of the gallbladder in patients with acute acalculous cholecystitis by transpapillary endoscopic cholecystotomy. Gastrointest Endosc 1993; 39: 645-651
[16] Shrestha R, Bilir BM, Everson GT et al. Endoscopic stenting of gallbladder for symptomatic cholelithiasis in patients with end-stage liver disease awaiting orthotopic liver transplantation. Am J Gastroenterol 1996; 91: $595-598$

[17] Schlenker C, Trotter JF, Shah RJ et al. Endoscopic gallbladder stent placement for treatment of symptomatic cholelithiasis in patients with end-stage liver disease. Am J Gastroenterol 2006; 101: 278-283

[18] Pannala R, Petersen BT, Gostout C] et al. Endoscopic transpapillary gallbladder drainage: 10-year single center experience. Minerva Gastroenterol Dietol 2008; 54: 107 - 113

[19] Inoue T, Okumura F, Kachi K et al. Long-term outcomes of endoscopic gallbladder stenting in high-risk surgical patients with calculous cholecystitis. Gastrointest Endosc 2016; 83: 905-913

[20] Hatanaka T, Itoi T, Ijima M et al. Efficacy and safety of endoscopic gallbladder stenting for acute cholecystitis in patients with concomitant unresectable cancer. Intern Med 2016; 55: 1411 - 1417

[21] Maekawa S, Nomura R, Murase T et al. Endoscopic gallbladder stenting for acute cholecystitis: a retrospective study of 46 elderly patients aged 65 years or older. BMC Gastroenterol 2013; 13: 65

[22] Yokoe M, Takada T, Strasberg SM et al. TG13 diagnostic criteria and severity grading of acute cholecystitis. J Hepatobiliary Pancreat Sci 2013; 20: $35-46$

[23] Itoi T, Coelho-Prabhu N, Baron TH. Endoscopic gallbladder drainage for management of acute cholecystitis. Gastrointest Endosc 2010; 71: $1038-1045$

[24] Khan MA, Atiq O, Kubiliun N et al. Efficacy and safety of endoscopic gallbladder drainage in acute cholecystitis: Is it better than percutaneous gallbladder drainage? Gastrointest Endosc 2017; 85: 76-87

[25] Jang JW, Lee SS, Song T] et al. Endoscopic ultrasound-guided transmural and percutaneous transhepatic gallbladder drainage are comparable for acute cholecystitis. Gastroenterology 2012; 142: 805 811

[26] Kedia P, Sharaiha RZ, Kumta NA et al. Endoscopic gallbladder drainage compared with percutaneous drainage. Gastrointest Endosc 2015; 82: $1031-1036$

[27] Tsuyuguchi T, Takada T, Kawarada Y et al. J Hepatobiliary Pancreat Surg. 2007; 14: $46-51$

[28] Cotton PB, Eisen GM, Aabakken L et al. A lexicon for endoscopic adverse events: report of an ASGE workshop. Gastrointest Endosc 2010; 71: $446-454$

[29] Itoi T, Kawakami H, Katanuma A et al. Endoscopic nasogallbladder tube or stent placement in acute cholecystitis: a preliminary prospective randomized trial in Japan. Gastrointest Endosc 2015; 81: $111-118$ 